OPEN ACCESS

Edited by:

Xiang Zhang,

Sun Yat-sen University, China

Reviewed by:

Fajin Dong,

Jinan University, China

Ning Mao,

Peking University People's Hospital,

China

*Correspondence:

Zhanguo Sun

yingxiangszg@163.com

$$
\begin{array}{r}
\text { Specialty section: } \\
\text { This article was submitted to } \\
\text { Breast Cancer, } \\
\text { a section of the journal } \\
\text { Frontiers in Oncology }
\end{array}
$$

Received: 30 November 2021

Accepted: 07 January 2022

Published: 31 January 2022

Citation:

Wang W, Zhang X, Zhu L, Chen Y,

Dou W, Zhao F, Zhou Z and Sun Z

(2022) Prediction of Prognostic

Factors and Genotypes in

Patients With Breast Cancer

Using Multiple Mathematical Models of MR Diffusion Imaging.

Front. Oncol. 12:825264.

doi: 10.3389/fonc.2022.825264

\section{Prediction of Prognostic Factors and Genotypes in Patients With Breast Cancer Using Multiple Mathematical Models of MR Diffusion Imaging} Weiwei Wang ${ }^{1}$, Xindong Zhang ${ }^{1}$, Laimin Zhu ${ }^{1}$, Yueqin Chen ${ }^{1}$, Weiqiang Dou ${ }^{2}$, Fan Zhao ${ }^{1}$,
Zhe Zhou ${ }^{1}$ and Zhanguo Sun ${ }^{1 *}$

${ }^{1}$ Department of Medical Imaging, Affiliated Hospital of Jining Medical University, Jining, China,

${ }^{2}$ MR Research, GE Healthcare, Beijing, China

Purpose: To explore the clinical value of apparent diffusion coefficient (ADC), intravoxel incoherent motion (IVIM), and diffusion kurtosis imaging (DKI) based on diffusion-weighted MRI (DW-MRI) for predicting genotypes and prognostic factors of breast cancer.

Materials and Methods: A total of 227 patients with breast cancer confirmed by pathology were reviewed retrospectively. Diffusion-weighted imaging (DWI), IVIM, and DKI were performed in all patients. The corresponding ADC, true diffusion coefficient (D), perfusion-related diffusion coefficient $\left(D^{*}\right)$, perfusion fraction $(f)$, mean diffusion rate $(M D)$, and mean kurtosis value (MK) were measured. Multivariate logistic regression analysis and receiver operating characteristic $(\mathrm{ROC})$ curve were used to analyze the diagnostic efficacy in predicting the Nottingham prognostic index (NPI), the expression of antigen Ki-67, and the molecular subtypes of breast cancer. The nomogram of the combined genotype-prediction model was established based on the multivariate logistic regression model results.

Results: $D^{*}$ and MK values were significantly higher in the high-grade Nottingham group $(\mathrm{NPI} \geq 3.4)$ than the low-grade Nottingham group $(\mathrm{NPI}<3.4)(\mathrm{p}<0.01)$. When $\mathrm{D}^{*} \geq 30.95 \times$ $10^{-3} \mathrm{~mm}^{2} / \mathrm{s}$ and $\mathrm{MK} \geq 0.69$, the NPI tended to be high grade (with areas under the curve (AUCs) of 0.712 and 0.647 , respectively). The combination of $D^{*}$ and $M K$ demonstrated the highest AUC of 0.734 in grading NPI with sensitivity and accuracy of $71.7 \%$ and $77.1 \%$, respectively. Additionally, higher $D^{*}, \mathrm{f}$, and MK and lower ADC and D values were observed in the high Ki-67 than low Ki-67 expression groups ( $p<0.05)$. The AUC of the combined model $\left(D+D^{*}+f+M K\right)$ was 0.755 , being significantly higher than that of single parameters $(Z=2.770 \sim 3.244, p=0.001 \sim 0.006)$ in distinguishing high from low Ki-67 expression. $D^{*}$ and $f$ values in the Luminal $A$ subtype were significantly lower than in other subtypes $(p<$ $0.05)$. Luminal $B$ showed decreased $D$ value compared with other subtypes $(p<0.05)$. The HER-2-positive subtype demonstrated increased ADC values compared with the Luminal B subtype $(p<0.05)$. Luminal A/B showed significantly lower $D, D^{*}, M D$, and $M K$ than the non-Luminal subtypes $(p<0.05)$. The combined model $\left(D+D^{*}+M D+M K\right)$ showed an AUC of 0.830 in diagnosing the Luminal and non-Luminal subtypes, which is significantly 
higher than that of a single parameter $(Z=3.273 \sim 4.440, p<0.01) . f \geq 54.30 \%$ [odds ratio $(O R)=1.038, p<0.001]$ and $M K \geq 0.68(O R=24.745, p=0.012)$ were found to be significant predictors of triple-negative subtypes. The combination of $f$ and MK values demonstrated superior diagnostic performance with AUC, sensitivity, specificity, and accuracy of $0.756,67.5 \%, 77.5 \%$, and $82.4 \%$, respectively. Moreover, as shown in the calibration curve, strong agreements were observed between nomogram prediction probability and actual findings in the prediction of genotypes $(p=0.22,0.74)$.

Conclusion: DWI, IVIM, and DKI, as MR diffusion imaging techniques with different mathematical models showed potential to identify the prognosis and genotype of breast cancer. In addition, the combination of these three models can improve the diagnostic efficiency and thus may contribute to opting for an appropriate therapeutic approach in clinic treatment.

Keywords: breast neoplasms, magnetic resonance imaging, diffusion-weighted imaging, intravoxel incoherent motion, diffusion kurtosis imaging

\section{INTRODUCTION}

Breast cancer is the most common malignancy among women (1). The management and overall survival of breast cancer are highly individualized and routinely based upon prognostic factors, such as the Nottingham prognostic index (NPI), the antigen Ki-67, and molecular expression signatures $(2,3)$. The NPI is the most validated system in breast cancer with the least interobserver variability currently (4). A higher Nottingham grade is associated with shorter survival and early recurrence, irrespective of tumor size, hormone receptor status, or lymph node metastasis status $(5,6)$. The Ki-67 index, reflecting the extent of proliferative activity, is a reliable identifier of more aggressive breast cancer and is associated with high risk for metastasis or recurrence, worse prognosis, and decreased survival (7). Furthermore, preoperative genotyping of breast cancer is essential because it may predict neoadjuvant chemotherapy responsiveness and allow optimized strategies for patient-tailored therapy. The Luminal A subtype is less responsive to chemotherapy, whereas the Luminal B subtype is responsive not only to chemotherapy but also to endocrine treatment or molecular-targeted therapy. The HER-2-positive subtype is insensitive to endocrine therapy but sensitive to targeted drugs such as trastuzumab therapy $(8,9)$. Triplenegative breast cancer (TNBC) lacks expressions of all three receptors (ER, $\mathrm{PR}$, and HER-2) and is known to have a more aggressive clinical course and poorer outcomes (10,11). However, both the prognostic factors and genotypes need to be obtained by biopsy or surgery.

MRI has a greater sensitivity than mammography or ultrasound in the diagnosis of breast cancer (12). Both dynamic contrast-enhanced MRI (DCE-MRI) and diffusion-weighted imaging (DWI) can detect the microscopic features of tumors. However, DCE-MRI requires intravenous contrast media administration; thus, it is not suitable to be used in patients with renal dysfunction. Moreover, as a semiquantitative analysis, time-signal intensity curve (TIC) assessment was reported with a low specificity in benign and malignant breast lesions (13). DWI with apparent diffusion coefficient (ADC) is routinely used in breast diagnosis, but the reported diagnostic reliability is still controversial, mainly due to inaccurate depiction of water molecule diffusion with the Gaussian model and influence of microcirculation perfusion (14). To address these two issues, an extended diffusion model of diffusion kurtosis imaging (DKI) reflects non-Gaussian diffusive motions of water in biologic tissues and has the potential to characterize the tissue heterogeneity and the interaction between water molecules and adjacent tissues (15). Meanwhile, intravoxel incoherent motion (IVIM) with multiple b-values, as another advanced diffusion model, allows the usage of a bi-exponential model to derive fast and slow diffusion parameters, aiming to separate diffusion from perfusion behaviors and better reflect the internal situation of tumors (16).

So far, multiple diffusion imaging techniques, including DWI, IVIM, or DKI, have been applied to evaluate the diagnostic value for prognostic factors and genotypes previously (17-19). However, to our best knowledge, no study was implemented to systematically apply these three techniques with individual mathematical models for comparison and to investigate the potential of the combined model in discriminating the prognostic factors and genotypes of breast cancer.

This study aimed to quantitatively compare the diagnostic performance of DWI, IVIM, DKI, and combined models for discriminating the prognostic factors and genotypes of breast cancer.

\section{MATERIALS AND METHODS}

\section{Patients}

From January 2019 to August 2021, 279 patients with breast cancer, confirmed by pathological examination, were recruited. The inclusion criteria were as follows: a) no contraindications to MRI examination; and b) all patients underwent routine MRI 
and multi-b-value DWI images. The exclusion criteria were as follows: a) receiving surgery, biopsy, or chemoradiotherapy before the examination; b) unsatisfactory imaging quality; c) time interval between MRI and surgery or biopsy was more than 2 weeks; and d) breast lesion with a size of less than $5 \mathrm{~mm}$. Figure 1 shows the flow diagram of the recruitment process. Finally, 227 patients were included in this study.

\section{MRI Acquisition}

A 3.0-T MR scanner (Discovery MR 750W, GE Medical Systems, Chicago, IL, USA) with a 16-channel phased-array coil specific for breast imaging was used for all MRI experiments. All patients were scanned in the prone position, with breasts naturally suspended in the coil. Premenopausal patients were examined in the second week of the menstrual cycle. The routine scan sequences were performed as follows: a transverse axial fast spinecho T1-weighted imaging (FSE-T1WI) sequence [repetition time $(\mathrm{TR}) /$ echo time $(\mathrm{TE})=420 / 10 \mathrm{~ms}$, field of view $(\mathrm{FOV})=$ $320 \mathrm{~mm} \times 288 \mathrm{~mm}$, slice thickness $/$ gap $=5 / 1 \mathrm{~mm}]$ and an axial fat-suppressed fast-recovery fast spin-echo T2-weighted imaging $($ FRFSE-T2WI) sequence $(\mathrm{TR} / \mathrm{TE}=6,000 / 88 \mathrm{~ms}, \mathrm{FOV}=320 \times$ $288 \mathrm{~mm}$, slice thickness/gap $=5 / 1 \mathrm{~mm})$. DWI, IVIM, and DKI were acquired before contrast injection using spin-echo echoplanar imaging sequence (SE-EPI). The parameters of DWI were as follows: TR/TE $=3,600 / 73 \mathrm{~ms}$, the excitations $(\mathrm{NEX})=2$, and b-values of 0 and $1,000 \mathrm{~s} / \mathrm{mm}^{2}$. IVIM was performed with the following parameters: $\mathrm{TR} / \mathrm{TE}=2,500 / 90 \mathrm{~ms}$, matrix $=128 \times 128$ $\mathrm{mm}$. Thirteen b-values $(0,20,30,50,70,100,150,200,500,700$, $1,000,1,500,2,000 \mathrm{~s} / \mathrm{mm}^{2}$ ) were used in three orthogonal directions. As the b-value increased, the number of NEX also increased from 1 to 6 to ensure a sufficient image signal-to-noise ratio (SNR). The total acquisition time for IVIM was 6 min $40 \mathrm{~s}$. DKI was obtained at b-values of $0,1,000$, and $2,000 \mathrm{~s} / \mathrm{mm}^{2}$. Fifteen diffusion gradient directions were set separately at bvalues of 1,000 and $2,000 \mathrm{~s} / \mathrm{mm}^{2}$. Other scan parameters were $\mathrm{TR} / \mathrm{TE}=5,000 / 90 \mathrm{~ms}$, matrix $=128 \times 128, \mathrm{NEX}=2$, and scan time $=5$ min $55 \mathrm{~s}$. The section thickness/gap and FOV of DWI, IVIM, and DKI were copied from the FRFSE-T2WI sequence.

\section{Patients underwent MR examination before surgery and} confirmed breast cancer postoperative pathology $(n=279)$

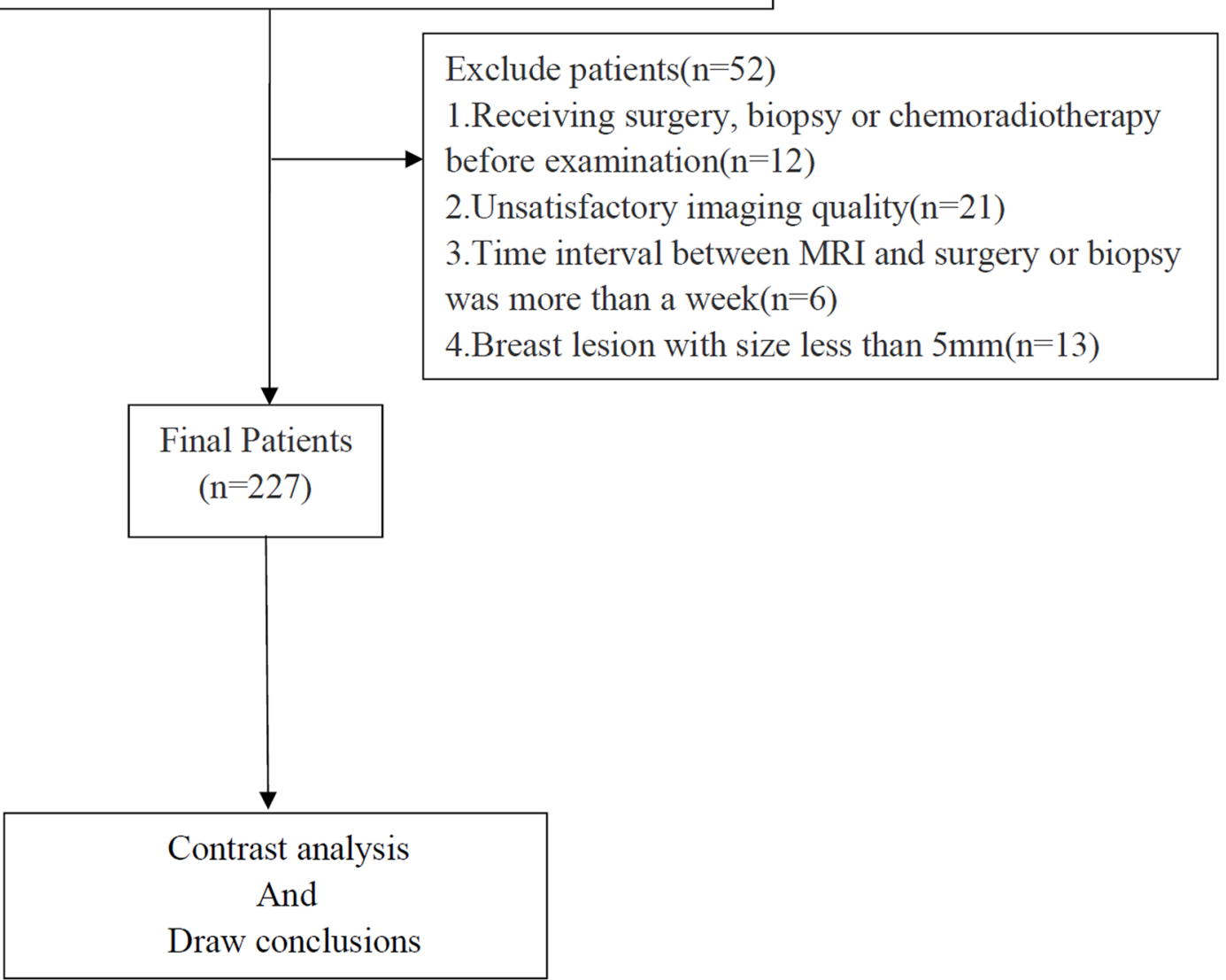

FIGURE 1 | Flowchart of the enrolled patients. 


\section{Data Analysis}

All images were transferred to Advantage Workstation (version AW 4.6, GE Medical Systems) for post-processing. Acquired DWI, IVIM, and DKI data were processed by vendor-provided software (Function tool MADC and DKI software; GE Healthcare) to acquire corresponding parametric maps.

For DWI, ADC maps were generated on a pixel-by-pixel basis according to a mono-exponential model: $\mathrm{S}_{\mathrm{b}} / \mathrm{S}_{0}=\exp (-\mathrm{b} \cdot \mathrm{ADC})$, where $b$ is the diffusion factor, and $S_{b}$ and $S_{0}$ are the signal intensities with diffusion factors of 1,000 and $0 \mathrm{~s} / \mathrm{mm}^{2}(20)$.

IVIM-derived parameters were calculated based on the following bi-exponential model: $\mathrm{S}_{\mathrm{b}} / \mathrm{S}_{0}=(1-\mathrm{f}) \times \exp (-\mathrm{b} \times \mathrm{D})+$ $\mathrm{f} \times \exp \left[-\mathrm{b} \times\left(\mathrm{D}^{\star}+\mathrm{D}\right)\right]$, where $\mathrm{S}_{\mathrm{b}}$ is the diffusion-weighted signal at a certain b-value; $S_{0}$ is the signal without diffusion weighting at $\mathrm{b}=0$; $\mathrm{D}$, true diffusion coefficient, represents pure water molecular diffusion in tissues; $\mathrm{D}^{*}$, pseudo-diffusion coefficient, a fast component of diffusion, reflects the incoherent movements of microvascular blood within the voxel; and f, perfusion fraction, represents the volume fraction of random microcirculation over the total incoherent signal in each voxel (21).

DKI parameters were calculated using the following equation: $S_{b}=S_{0} \cdot \exp \left(-b^{2} \cdot D^{2}+b \cdot D^{2} \cdot K / 6\right)$, where $S_{0}$ and $S_{b}$ represent the signal intensity (SI) under different b-values $\left(0 \mathrm{~s} / \mathrm{mm}^{2}\right.$ or other values); $\mathrm{K}$ (arbitrary units) indicates kurtosis and represents the degree of deviation from the Gaussian distribution; and D $\left(\times 10^{-3}\right.$ $\mathrm{mm}^{2} / \mathrm{s}$ ) indicates diffusivity and represents the diffusion coefficient corrected for non-Gaussian bias (22).

The region of interest (ROI) was delineated on the grayscale map with a b-value of $1,000 \mathrm{~s} / \mathrm{mm}^{2}$ (12), and then, the pseudocolor images of the IVIM and DKI parameters were merged with the grayscale map by using 3D SynchroView (GE Healthcare) (Figure 2). The ROI included as much of the solid region of the tumor as possible, while regions with large blood vessels, necrosis, or hemorrhage were avoided. For patients with multicentric or multifocal tumors, only the tumors with the largest diameter were analyzed. For the non-mass lesions, the ROI was placed on the representative solid slice of the tumor by using the plain scan and contrast-enhanced sequence as references. The ROI was delineated by two independent radiologists (LZ and WW with 15 and 5 years of experience, respectively). In order to reduce the measurement error caused by the bias of ROI selection, the maximum layer of the lesion, and the upper and lower consecutive levels were measured three times. Then, the corresponding average value was calculated for data analysis.

\section{Pathological Evaluation}

All patients underwent breast-conserving surgery or mastectomy, while 64 patients received additional biopsy before operation. Tumor size, axillary node status, histologic type, histologic grade, and lymphovascular invasion status were determined based on surgically excised specimens. The pathological evaluation of the surgically resected specimens was performed by two pathologists (with 4 and 12 years of experience) independently. NPI was calculated according to the following formula: NPI $=$ size $(\mathrm{cm}) \times 0.2+$ lymph node staging
$(1-3)+$ histologic grade $(1-3)$. No metastatic lymph nodes is 1 point, $1-4$ nodes is 2 points, and more than 4 nodes is 3 points. Based on NPI scores, the low-grade (2.0-3.4 points) and highgrade (>3.41 points) groups were defined (23). Tumor subtypes were classified as Luminal A for ER or PR positive, HER-2 negative, and Ki-67 negative; as Luminal B for ER or PR positive, HER-2 positive, and Ki-67 positive; as HER-2 positive for ER and PR negative and HER-2 positive; and as triple negative (TN) for ER, PR, and HER-2 negative. ER and PR positivity were defined as $10 \%$ or with more positively stained nuclei in 10 high-power fields. Ki-67 labeling was defined as negative $(<14 \%)$ or positive $(\geq 14 \%)$. The intensity of HER-2 staining was semiquantitatively scored as $0,1+, 2+$, or $3+$. Tumors with a $3+$ score were classified as HER-2 positive, and tumors with 0 or $1+$ score were classified as HER-2 negative. In tumors with a $2+$ score, gene amplification with fluorescence in situ hybridization was used to determine HER-2 status (24).

\section{Statistical Analysis}

Statistical analyses were conducted using SPSS 25.0 (IBM Corporation, Armonk, NY, USA), MedCalc 19.5.1 (Ostend, Belgium), and R version 4.0.0 (http://www.r-project.org/). The interobserver consistency was assessed by inter-class correlation coefficients (ICCs). The interpretation of ICC values was defined as follows: $0.00-0.20$, poor agreement; $0.21-0.40$, fair agreement; 0.41-0.60, moderate agreement; $0.61-0.80$, good agreement; and 0.81-1.00, excellent agreement (25). The Kolmogorov-Smirnov test was used to determine whether two samples of measurement data were normally distributed. An independent-samples t-test was used to compare the $\mathrm{ADC}, \mathrm{D}, \mathrm{MD}$, and $\mathrm{MK}$ values between different prognostic factors. The Mann-Whitney $U$ test was used to detect the difference in $\mathrm{D}^{*}$ and $\mathrm{f}$ values between the different prognostic factors. Moreover, one-way ANOVA was used for multiple comparisons of $\mathrm{ADC}, \mathrm{D}, \mathrm{MD}$, and $\mathrm{MK}$ values between different genotypes; and the Kruskal-Wallis $\mathrm{H}$ test was performed to compare $\mathrm{D}^{*}$ values among different genotypes. Multivariate logistic regression analyses were used to identify independent factors. Receiver operating characteristic (ROC) curves were used to assess the diagnostic efficacy of each parameter or model in discriminating prognostic factors or genotypes, and the Delong test was used to determine whether the area under the curve (AUC) of each ROC was significantly different. $\mathrm{p}<0.05$ was considered statistically significant. A nomogram was developed based on the outcomes of multivariate logistic regression to predict the genotypes. And a calibration using bootstraps with 1,000 resamples for internal validation by comparing nomogram-predicted versus nomogram-observed response probability was done as well as the Hosmer-Lemeshow goodness-of-fit test.

\section{RESULTS}

\section{Clinical and Pathological Characteristics}

The average age of the 227 patients was $50.8 \pm 10.3$ years (range 27-86). The histological types included 206 invasive ductal 


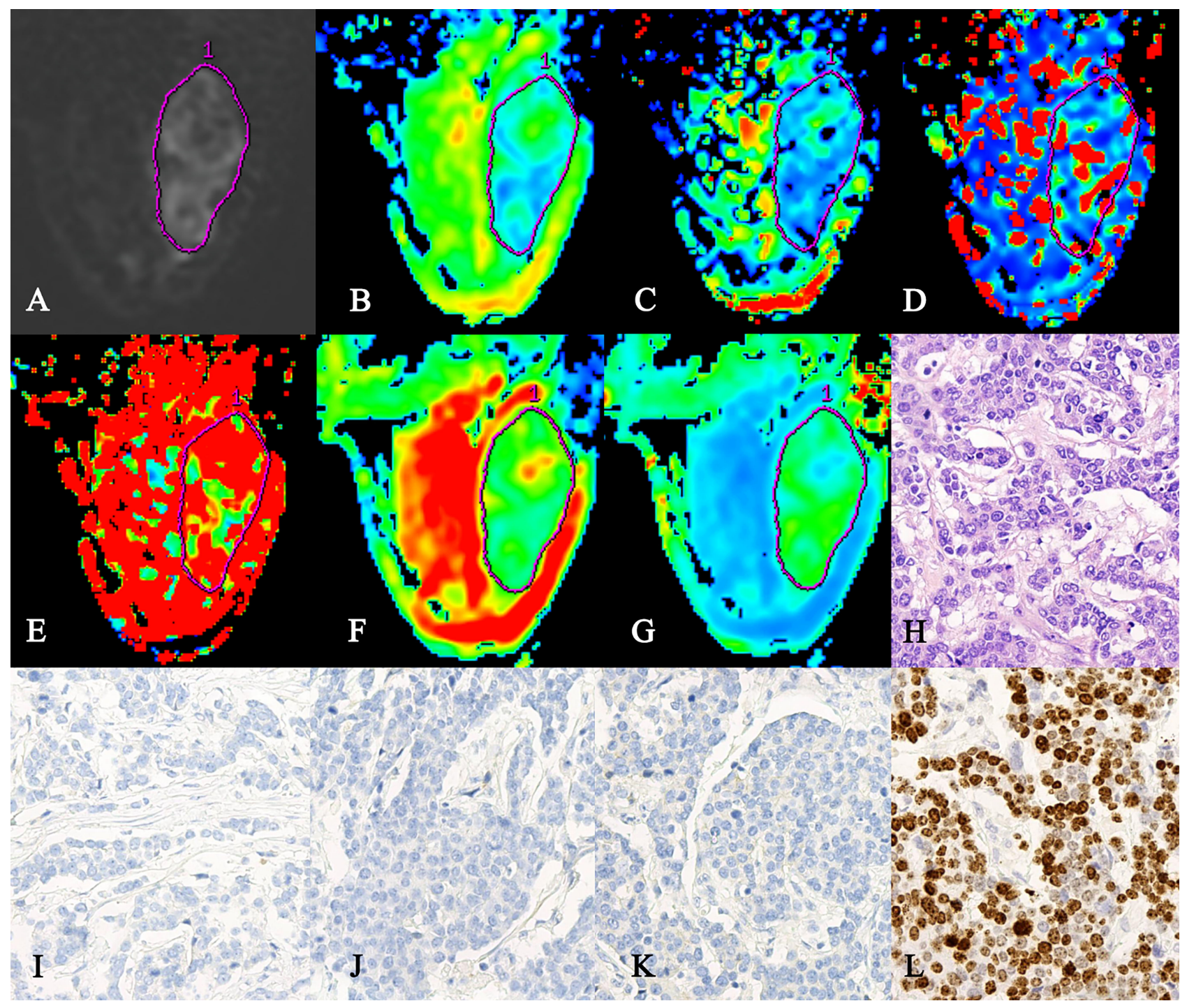

FIGURE 2 | A 47-year-old female patient with triple-negative cancer in the right breast. (A) ROI was set on the grayscale map with b-value of 1,000 s/mm². (B-G) The pseudo-colored maps of the ADC, D, D*, f, MD, and MK. ADC $=1.05 \times 10^{-3} \mathrm{~mm}^{2} / \mathrm{s}, \mathrm{D}=0.92 \times 10^{-3} \mathrm{~mm}^{2} / \mathrm{s}, \mathrm{D}^{\star}=41.8 \times 10^{-3} \mathrm{~mm}^{2} / \mathrm{s}, \mathrm{f}=81.1 \%, \mathrm{MD}=2.58 \times 10^{-3}$ $\mathrm{mm}^{2} / \mathrm{s}$, and $\mathrm{MK}=0.774$. (H) H\&E staining of the invasive breast ductal carcinoma ( $\left.\times 200\right)$. (I-L) Immunohistochemistry staining for the ER (I), PR (J), HER-2 (K), and $\mathrm{Ki}-67$ (L) in the invasive breast ductal carcinoma. ROI, region of interest; ER, estrogen receptor; PR, progesterone receptor.

carcinomas (90.7\%), 7 ductal carcinoma in situ (3.1\%), 4 invasive lobular carcinomas (1.8\%), 3 mucinous carcinomas (1.3\%), 3 invasive ropapillary carcinomas $(1.3 \%), 3$ medullary carcinoma (1.3\%), and 1 cribriform carcinoma (0.5\%). Of the 227 lesions, $151(66.5 \%)$ were classified as Luminal subtype, $36(15.9 \%)$ as HER-2-positive subtype, and $40(17.6 \%)$ as TNBC subtype (Table $\mathbf{1}$ ).

\section{Interobserver Agreement}

The ICCs between the two radiologists were 0.878 [95\% CI: $0.842-$ 0.906], 0.820 (95\% CI: 0.766-0.861), 0.908 (95\% CI: 0.880-0.929), 0.892 (95\% CI: $0.860-0.917), 0.870$ (95\% CI: $0.832-0.900)$, and 0.886 (95\% CI: $0.852-0.912$ ) for $\mathrm{ADC}, \mathrm{D}, \mathrm{D}^{*}, \mathrm{f}, \mathrm{MD}$, and $\mathrm{MK}$ measurements, respectively, indicating an excellent interobserver agreement.

\section{Diagnostic Performance of Diffusion- Weighted Imaging-, Intravoxel Incoherent Motion-, and Diffusion Kurtosis Imaging- Derived Parameters in Differentiating Prognostic Factors of Breast Cancer}

The $\mathrm{D}^{*}$ and $\mathrm{MK}$ values were significantly higher in tumors of the high-grade Nottingham group (NPI $\geq 3.4$ ) than those of the lowgrade Nottingham group $(\mathrm{NPI}<3.4)(\mathrm{p}<0.01)$. The $\mathrm{D}^{\star}, \mathrm{f}$, and $\mathrm{MK}$ values were higher and the $\mathrm{ADC}$ and $\mathrm{D}$ values were lower in 
TABLE 1 | Clinicopathological characteristics of the included patients.

\begin{tabular}{|c|c|}
\hline Characteristics & Data \\
\hline Age (years), mean \pm SD & $50.8 \pm 10.3$ \\
\hline Mean tumor size $(\mathrm{cm})$, mean \pm SD & $2.56 \pm 1.22$ \\
\hline \multicolumn{2}{|l|}{ Histologic grade, $\mathrm{n}(\%)$} \\
\hline 1 & $16(7.0 \%)$ \\
\hline 2 & 99 (43.6\%) \\
\hline 3 & $112(49.4 \%)$ \\
\hline \multicolumn{2}{|l|}{ Histological type, n (\%) } \\
\hline Invasive ductal carcinoma & $206(90.7 \%)$ \\
\hline Non-invasive ductal carcinoma & $21(9.3 \%)$ \\
\hline \multicolumn{2}{|l|}{ Nodal status, n (\%) } \\
\hline Negative (-) & $95(41.9 \%)$ \\
\hline Positive (+) & $132(58.1 \%)$ \\
\hline \multicolumn{2}{|l|}{ ER, n (\%) } \\
\hline Negative (-) & 77 (33.9\%) \\
\hline Positive (+) & $150(66.1 \%)$ \\
\hline \multicolumn{2}{|l|}{ PR, n (\%) } \\
\hline Negative (-) & 95 (41.9\%) \\
\hline Positive (+) & $132(58.1 \%)$ \\
\hline \multicolumn{2}{|l|}{ HER-2, n (\%) } \\
\hline Negative (-) & $107(47.1 \%)$ \\
\hline Positive (+) & $120(52.9 \%)$ \\
\hline \multicolumn{2}{|l|}{ Ki-67, n (\%) } \\
\hline Negative (-) & $61(26.9 \%)$ \\
\hline Positive (+) & $166(73.1 \%)$ \\
\hline \multicolumn{2}{|l|}{ Genotypes, n (\%) } \\
\hline Luminal A & $29(12.8 \%)$ \\
\hline Luminal B & $122(53.7 \%)$ \\
\hline HER-2-positive & 36 (15.9\%) \\
\hline Triple-negative & $40(17.6 \%)$ \\
\hline
\end{tabular}

$E R$, estrogen receptor; $P R$, progesterone receptor.

the high Ki-67 expression group than in the low expression group ( $<$ 0.05) (Table 2 ).

\section{Diagnostic Efficiency of Diffusion- Weighted Imaging-, Intravoxel Incoherent Motion-, and Diffusion Kurtosis Imaging- Derived Parameters for Predicting Nottingham Prognostic Index and Ki-67}

According to the multivariate logistic regression, $\mathrm{D}^{\star}$ [odds ratio $(\mathrm{OR})=1.038, \mathrm{p}<0.001]$ and $\mathrm{MK}(\mathrm{OR}=24.745, \mathrm{p}=0.012)$ were found to be significant predictors of NPI. When $\mathrm{D}^{\star} \geq 30.95 \times 10^{-3}$ $\mathrm{mm}^{2} / \mathrm{s}$ and $\mathrm{MK} \geq 0.69$, the NPI tended to be high grade, and the AUCs were 0.712 and 0.647 , respectively. The combination of MK and $\mathrm{D}^{\star}$ demonstrated the highest sensitivity and accuracy of
$71.7 \%$ and $77.1 \%$, respectively. The AUC of the combined model $\left(\mathrm{D}^{*}+\mathrm{MK}\right)$ was significantly higher than that of $\mathrm{MK}(\mathrm{Z}=2.148, \mathrm{p}=$ 0.032), whereas there was no statistically significant difference from that of $D^{*}(Z=0.879, p=0.379)$ (Table 3 and Figure 3A).

$\mathrm{D}(\mathrm{OR}=1.623, \mathrm{p}=0.046), \mathrm{D}^{*}(\mathrm{OR}=0.972, \mathrm{p}=0.002), \mathrm{f}(\mathrm{OR}=$ $0.964, \mathrm{p}=0.003)$, and $\mathrm{MK}(\mathrm{OR}=0.066, \mathrm{p}=0.011)$ were independent factors in evaluating the Ki-67 expression status. When $\mathrm{D} \leq 0.68 \times 10^{-3} \mathrm{~mm}^{2} / \mathrm{s}, \mathrm{D}^{*} \geq 31.02 \times 10^{-3} \mathrm{~mm}^{2} / \mathrm{s}, \mathrm{f} \geq$ $34.75 \%$, and $\mathrm{MK} \geq 0.65, \mathrm{Ki}-67$ tended to have high expression. The differences in AUCs of D (0.625), $\mathrm{D}^{\star}(0.634), \mathrm{f}(0.638)$, and MK (0.657) were not statistically significant $(Z=0.074 \sim 0.705, p=$ $0.481 \sim 0.940)$. The AUC of the combined model $\left(\mathrm{D}+\mathrm{D}^{\star}+\mathrm{f}+\mathrm{MK}\right)$ was 0.755 , being significantly higher than that of each single parameter $(\mathrm{Z}=2.770 \sim 3.244, \mathrm{p}=0.001 \sim 0.006$ ) (Table 3 and Figure 3B).

\section{Diagnostic Performance of Diffusion- Weighted Imaging-, Intravoxel Incoherent Motion-, and Diffusion Kurtosis Imaging- Derived Parameters in Differentiating Molecular Subtypes of Breast Cancer}

The $\mathrm{D}^{\star}$ and $\mathrm{f}$ values in the Luminal A subtype were significantly lower than those of other subtypes $(\mathrm{p}<0.05)$. Luminal A also exhibited decreased D value as compared with the HER-2positive subtype $(\mathrm{p}<0.05)$. The $\mathrm{D}$ value in the Luminal $\mathrm{B}$ subtype was significantly lower than that of other subtypes ( $\mathrm{p}<0.05$ ). The Luminal B subtype exhibited decreased $\mathrm{D}^{*}$ and MD values compared with the HER-2-positive and triplenegative subtypes $(\mathrm{p}<0.05)$. The Luminal A/B subtypes (the Luminal subtypes) showed significantly lower $\mathrm{D}, \mathrm{D}^{*}, \mathrm{MD}$, and MK than the non-Luminal subtypes $(p<0.05)$. The HER-2positive subtype demonstrated increased ADC values compared with the Luminal B subtype ( $\mathrm{p}<0.05)$. Triple-negative subtypes exhibited increased $f$ value compared with the HER-2-positive and Luminal B subtypes ( $\mathrm{p}<0.05$ ) (Table 4 and Figure 4).

\section{Development, Validation, and Diagnostic Efficiency of the Genotype-Prediction Models of Breast Cancer}

According to the multivariate logistic regression, $\mathrm{D}(\mathrm{OR}=21.023$, $\mathrm{p}<0.001), \mathrm{D}^{*}(\mathrm{OR}=1.017, \mathrm{p}=0.025), \mathrm{MD}(\mathrm{OR}=1.057, \mathrm{p}=$ $0.001)$, and $\mathrm{MK}(\mathrm{OR}=24.745, \mathrm{p}<0.001)$ were independent factors in identifying the Luminal subtypes from the non-Luminal

TABLE 2 | Diagnostic performance of DWI, DKI, and IVIM parameters in different prognostic factors of breast cancer.

\begin{tabular}{|c|c|c|c|c|c|c|}
\hline \multirow[t]{2}{*}{ Parameters } & \multicolumn{2}{|c|}{ NPI } & \multirow[t]{2}{*}{ p-Value } & \multicolumn{2}{|c|}{ Ki-67 } & \multirow[t]{2}{*}{ p-Value } \\
\hline & High & Low & & $<14 \%$ & $\geq 14 \%$ & \\
\hline $\operatorname{ADC}\left(\times 10^{-3} \mathrm{~mm}^{2} / \mathrm{s}\right)$ & $0.99 \pm 0.35$ & $1.00 \pm 0.33$ & 0.884 & $1.08 \pm 0.34$ & $0.96 \pm 0.34$ & 0.025 \\
\hline $\mathrm{D}\left(\times 10^{-3} \mathrm{~mm}^{2} / \mathrm{s}\right)$ & $0.67 \pm 0.32$ & $0.71 \pm 0.33$ & 0.407 & $0.76 \pm 0.29$ & $0.65 \pm 0.33$ & 0.022 \\
\hline $\mathrm{D}^{*}\left(\times 10^{-3} \mathrm{~mm}^{2} / \mathrm{s}\right)$ & $39.98 \pm 26.22$ & $23.89 \pm 16.99$ & $<0.001^{\star}$ & $26.77 \pm 15.97$ & $39.70 \pm 27.30$ & $<0.001^{\star}$ \\
\hline$f(\%)$ & $40.17 \pm 16.05$ & $37.93 \pm 14.77$ & $0.346^{\star}$ & $34.77 \pm 14.18$ & $41.44 \pm 15.97$ & $0.003^{*}$ \\
\hline $\mathrm{MD}\left(\times 10^{-3} \mathrm{~mm}^{2} / \mathrm{s}\right)$ & $2.43 \pm 0.76$ & $2.45 \pm 0.74$ & 0.868 & $2.46 \pm 0.79$ & $2.39 \pm 0.66$ & 0.500 \\
\hline MK & $0.74 \pm 0.23$ & $0.63 \pm 0.19$ & 0.001 & $0.63 \pm 0.17$ & $0.75 \pm 0.23$ & $<0.001$ \\
\hline
\end{tabular}

NPI, Nottingham prognostic index; DWI, diffusion-weighted imaging; DKI, diffusion kurtosis imaging; IVIM, intravoxel incoherent motion.

*Mann-Whitney $U$ test. 
TABLE 3 | Diagnostic efficiency of the IVIM and DKI models for predicting Nottingham index and Ki-67.

\begin{tabular}{|c|c|c|c|c|c|c|}
\hline Parameters & AUC & $95 \% \mathrm{Cl}$ & Cutoff & Sensitivity (\%) & Specificity (\%) & Accuracy (\%) \\
\hline \multicolumn{7}{|l|}{ Nottingham index } \\
\hline$D^{*}$ & 0.712 & $0.635 \sim 0.789$ & $30.95 \times 10^{-3} \mathrm{~mm}^{2} / \mathrm{s}$ & 64.9 & 81.1 & 76.7 \\
\hline MK & 0.647 & $0.563 \sim 0.730$ & 0.69 & 59.2 & 66.0 & 73.2 \\
\hline Combined model & 0.734 & $0.672 \sim 0.791$ & $:$ & 71.7 & 65.5 & 77.1 \\
\hline \multicolumn{7}{|l|}{ Ki-67 } \\
\hline D & 0.625 & $0.545 \sim 0.705$ & $0.68 \times 10^{-3} \mathrm{~mm}^{2} / \mathrm{s}$ & 60.7 & 65.7 & 71.8 \\
\hline$D^{\star}$ & 0.634 & $0.558 \sim 0.710$ & $31.02 \times 10^{-3} \mathrm{~mm}^{2} / \mathrm{s}$ & 61.8 & 68.9 & 72.6 \\
\hline$f$ & 0.638 & $0.813 \sim 0.913$ & $34.75 \%$ & 65.7 & 60.7 & 73.1 \\
\hline MK & 0.657 & $0.581 \sim 0.733$ & 0.65 & 68.1 & 59.0 & 73.5 \\
\hline Combined model & 0.755 & $0.694 \sim 0.809$ & : & 67.2 & 82.0 & 73.6 \\
\hline
\end{tabular}

IVIM, intravoxel incoherent motion; DKI, diffusion kurtosis imaging; AUC, area under the curve.

A

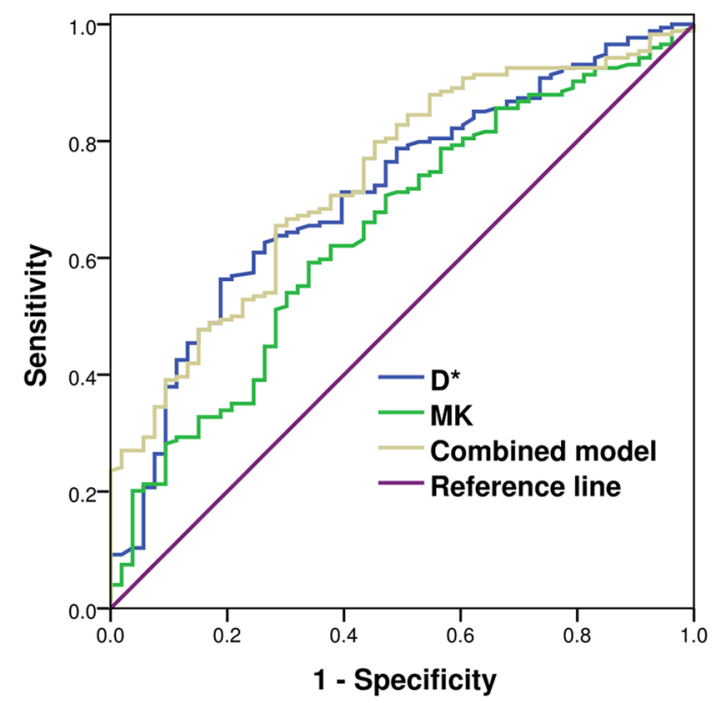

B

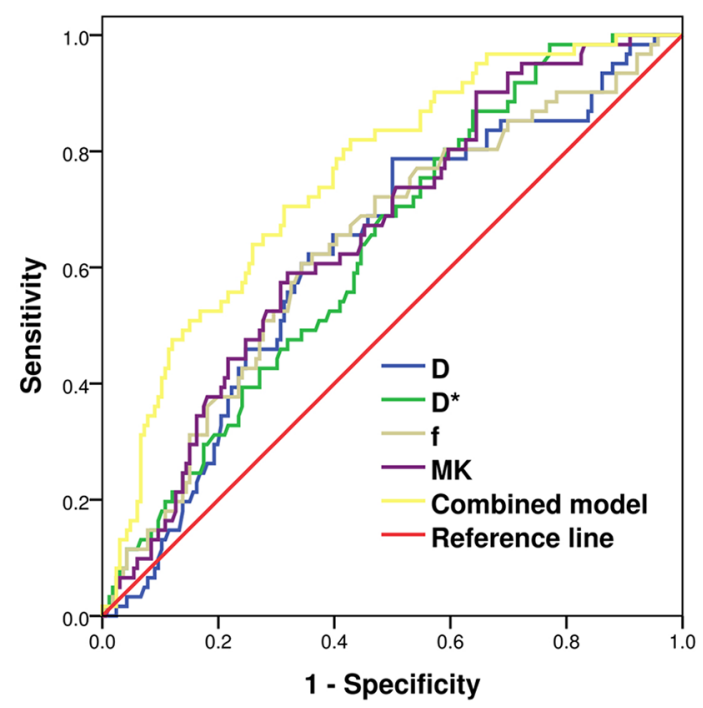

FIGURE 3 | ROC analysis of IVIM and DKI parameters in predicting Nottingham index (A) and Ki-67 (B) of breast cancer. ROC, receiver operating characteristic; IVIM, intravoxel incoherent motion; DKI, diffusion kurtosis imaging.

TABLE 4 | Diagnostic performance of DWI, DKI, and IVIM parameters in different genotypes of breast cancer.

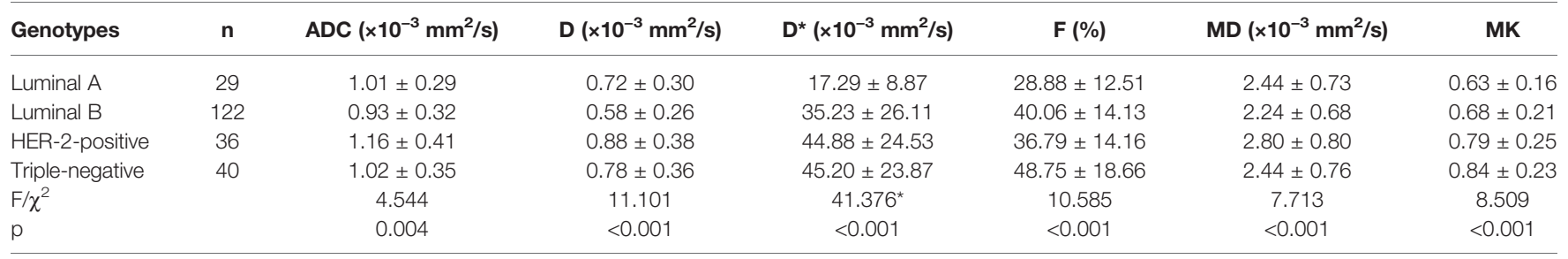

DWI, diffusion-weighted imaging; DKI, diffusion kurtosis imaging; IVIM, intravoxel incoherent motion.

*Kruskal-Wallis H test.

subtypes. The differences in AUCs were not statistically significant ( $\mathrm{Z}=0.164 \sim 0.826, \mathrm{p}=0.409 \sim 0.765)$. The AUC of the combined model $\left(\mathrm{D}+\mathrm{D}^{\star}+\mathrm{MD}+\mathrm{MK}\right)$ was 0.830 , which was significantly higher than that of each single parameter $(\mathrm{Z}=3.273 \sim 4.440, \mathrm{p}<$ 0.01) (Table 5 and Figure 5A). The nomogram model was thus generated by using these four independent factors as predictors (Figure 6A). As shown in the calibration curve, a good agreement was observed between nomogram prediction values and actual findings, and the Hosmer-Lemeshow test showed no significant difference $(\mathrm{p}=0.22)$ (Figure 7A).

$\mathrm{f} \geq 54.30 \%(\mathrm{OR}=1.038, \mathrm{p}<0.001)$ and $\mathrm{MK} \geq 0.68(\mathrm{OR}=$ $24.745, \mathrm{p}=0.012)$ were found to be significant predictors of triple-negative subtypes. The AUC of the combined model ( $\mathrm{f}+$ $\mathrm{MK})$ was significantly higher than that of $\mathrm{f}(\mathrm{Z}=2.521, \mathrm{p}=0.012)$, whereas there was no statistically significant difference from that of $\mathrm{MK}(\mathrm{Z}=1.645, \mathrm{p}=0.100)$ (Table 5 and Figure 5B). 


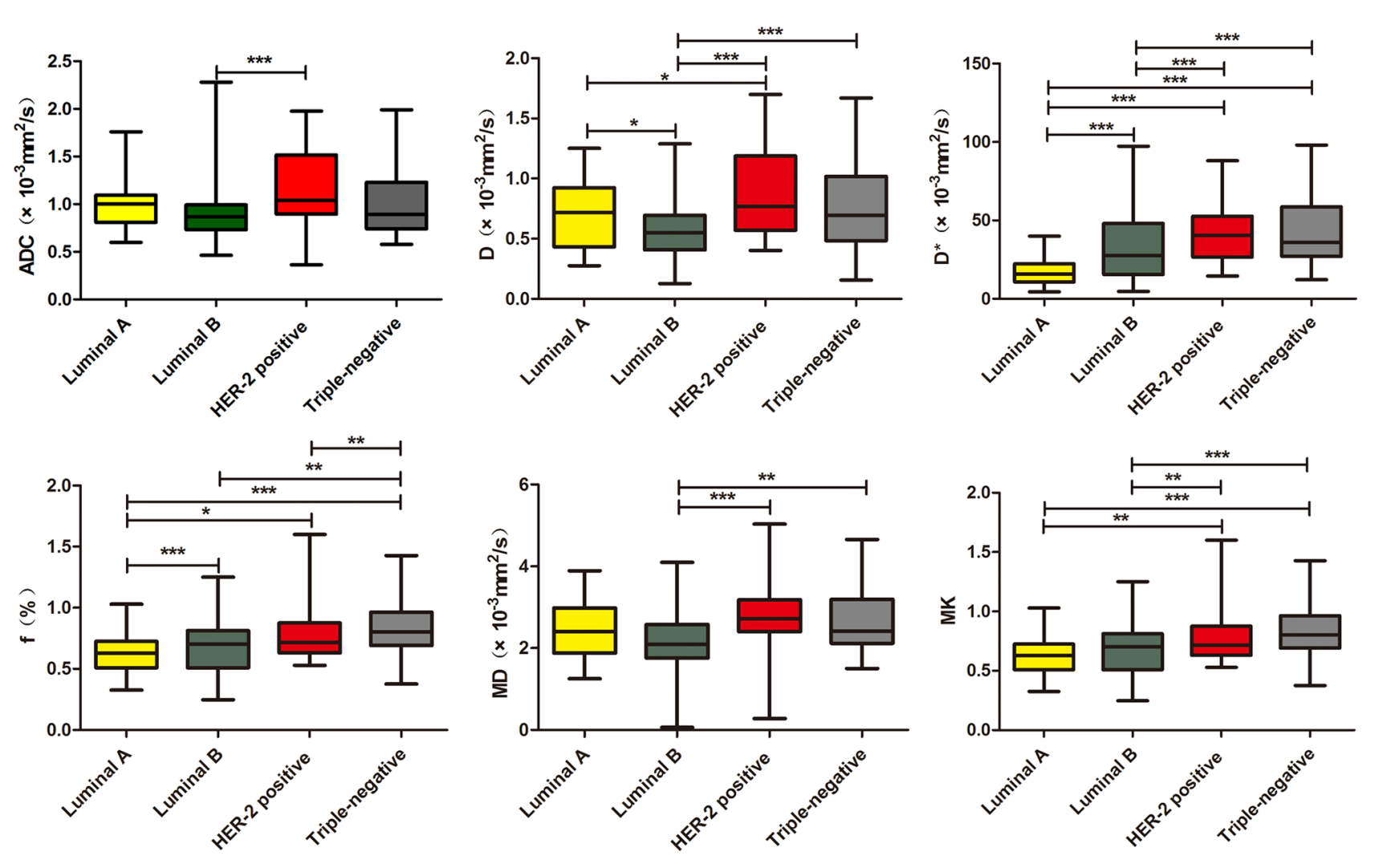

FIGURE 4 | Boxplots of ADC, D, D*, f, MD, and MK in differentiating genotypes of breast cancer. ${ }^{*} p<0.05,{ }^{* *} p<0.01,{ }^{* * *} \mathrm{p}<0.001$.

TABLE 5 | Diagnostic efficiency of the IVIM and DKI models for predicting molecular subtypes.

\begin{tabular}{|c|c|c|c|c|c|c|}
\hline Parameters & AUC & $95 \% \mathrm{Cl}$ & Cutoff & Sensitivity (\%) & Specificity (\%) & Accuracy (\%) \\
\hline \multicolumn{7}{|c|}{ Luminal A/B vs. non-Luminal } \\
\hline $\mathrm{D}$ & 0.676 & $0.602-0.750$ & $0.66 \times 10^{-3} \mathrm{~mm}^{2} / \mathrm{s}$ & 60.5 & 64.9 & 68.7 \\
\hline MD & 0.689 & $0.618-0.759$ & $2.27 \times 10^{-3} \mathrm{~mm}^{2} / \mathrm{s}$ & 73.7 & 57.6 & 67.0 \\
\hline MK & 0.666 & $0.594-0.738$ & 0.537 & 96.1 & 30.5 & 63.3 \\
\hline Combined model & 0.830 & $0.774-0.876$ & : & 73.2 & 87.4 & 80.2 \\
\hline \multicolumn{7}{|c|}{ Triple-negative vs. other genotypes } \\
\hline Combined model & 0.756 & $0.695-0.811$ & : & 67.5 & 77.5 & 82.4 \\
\hline
\end{tabular}

IVIM, intravoxel incoherent motion; DKI, diffusion kurtosis imaging; AUC, area under the curve.

A nomogram was established according to the multivariate logistic regression analysis findings (Figure 6B), and there was a good agreement between the bias-corrected curve and the ideal curve as shown in the calibration curve plot and the HosmerLemeshow test $(\mathrm{p}=0.74)$ (Figure 7B).

\section{DISCUSSION}

As shown in the results, the functional parameters of DWI, IVIM, and DKI revealed distinct values in different histopathological features and genotypes of breast cancer. For the prognostic factors, $\mathrm{D}^{*}$ and $\mathrm{MK}$ values were higher in the high- than low-level NPI group. The $\mathrm{f}, \mathrm{MK}$, and $\mathrm{D}^{*}$ values were higher and the $\mathrm{D}$ value was lower in the Ki-67-positive than Ki67-negative group. In terms of molecular subtypes, the $\mathrm{D}$ value of Luminal B was lower than that of other genotypes. $D^{*}$ and $f$ values of Luminal A were lower than those of other genotypes. With the combined model of $\mathrm{D}, \mathrm{D} *, \mathrm{MD}$, and $\mathrm{MK}$, the diagnostic efficiency of the Luminal subtypes was greatly improved. Compared with those of other genotypes, the $f$ and MK values of TN tumors were higher, and the combination of these two parameters can improve the prediction accuracy of TN tumors. 
A

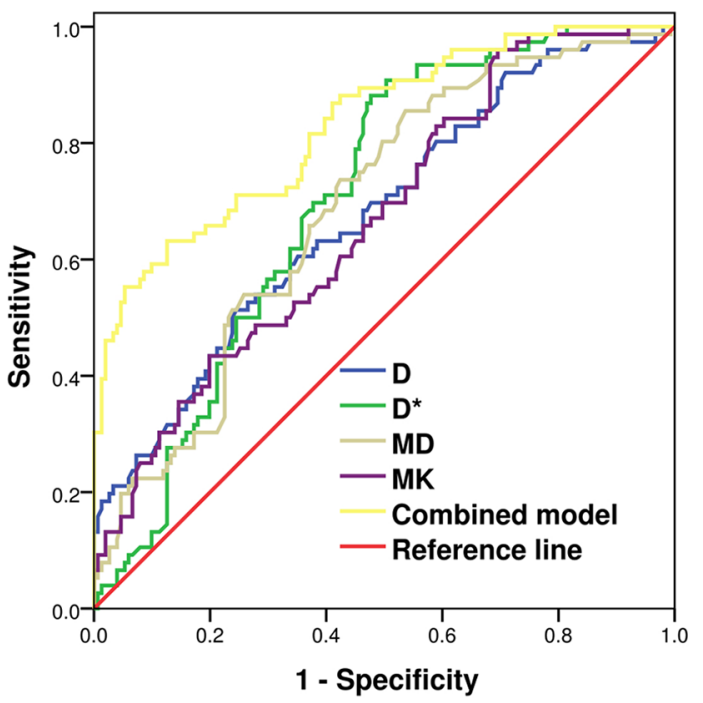

B

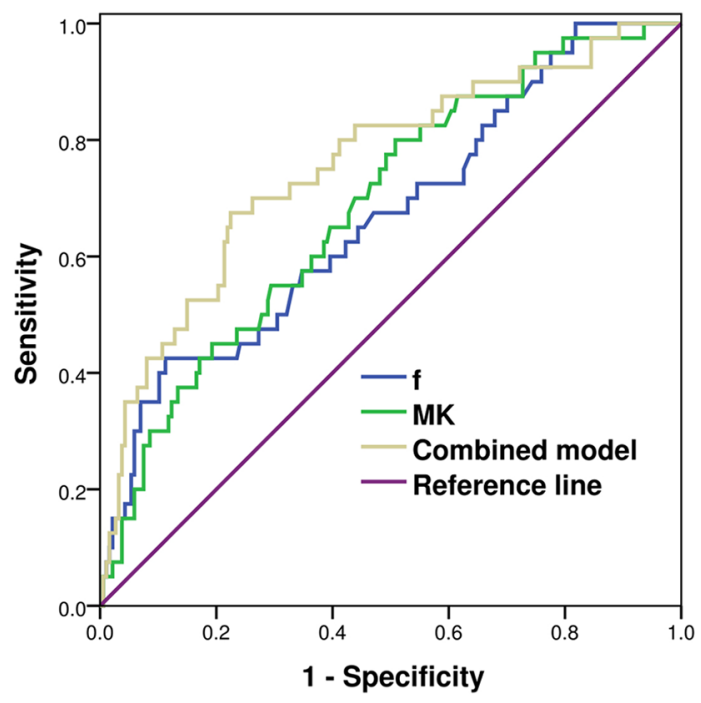

FIGURE 5 | ROC analysis of IVIM and DKI parameters in predicting Luminal subtypes (A) and triple-negative subtype of breast cancer (B). ROC, receiver operating characteristic; IVIM, intravoxel incoherent motion; DKI, diffusion kurtosis imaging.

A

Points

$10 \quad 10 \quad 20$

$20 \quad 30$

40

50

60

70

80

90 100

$\mathrm{D}\left(10^{-3} \mathrm{~mm}^{2} / \mathrm{s}\right)$

$D^{*}\left(10^{-3} \mathrm{~mm}^{2} / \mathrm{s}\right)$

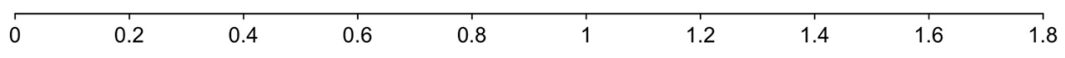

$\operatorname{MD}\left(10^{-3} \mathrm{~mm}^{2} / \mathrm{s}\right)$

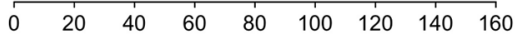

MK

Total Points

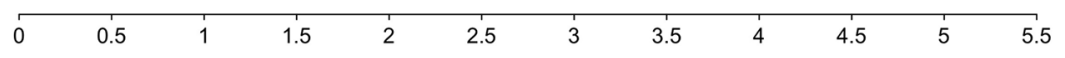

$\begin{array}{llllll}0.2 & 0.3 & 0.4 & 0.5 & 0.6 & 0.7\end{array}$

Risk

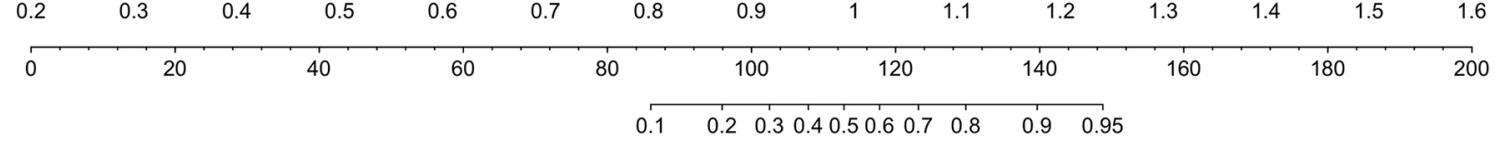

B

Points

0

$10 \quad 20$

30

40

50

60

70

80

90

100

$f(\%)$

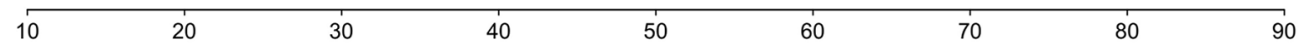

MK

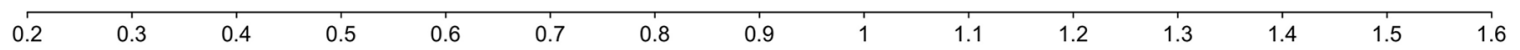

Total Points

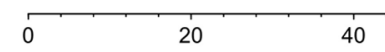

60

$80+100$

120

140

160

180

Risk

$\begin{array}{llllllll}0.1 & 0.2 & 0.3 & 0.4 & 0.5 & 0.6 & 0.7 & 0.8\end{array}$

FIGURE 6 | The nomogram for predicting the Luminal subtypes (A) and triple-negative subtype of breast cancer (B). 
A

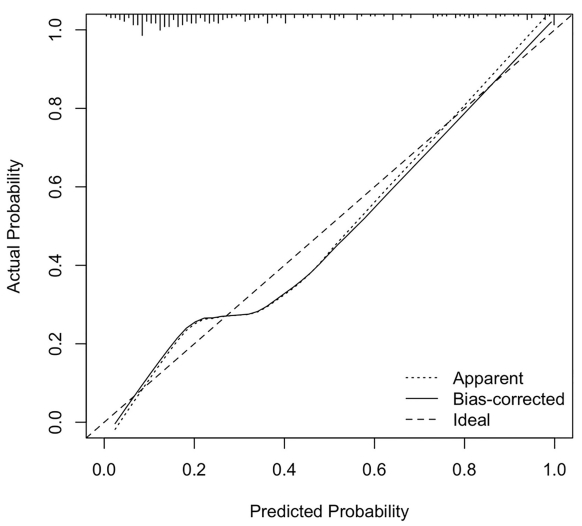

B

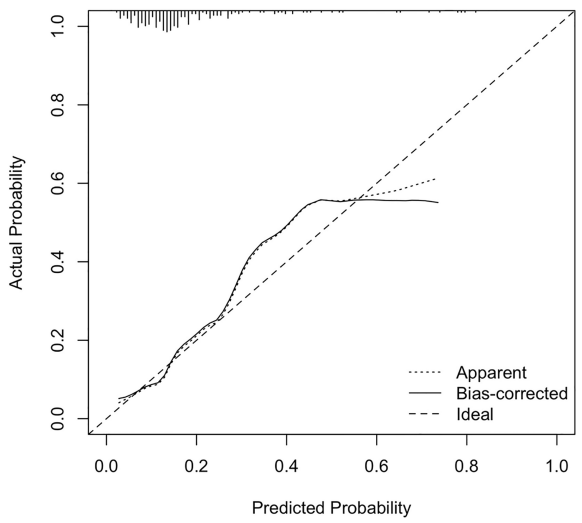

FIGURE 7 | Calibration plot for internal validation of the Luminal subtypes (A) and triple-negative subtype (B). The x-axis is the nomogram-predicted probability of genotypes. The $y$-axis is the actual probability. The dotted line represents an ideal standard curve; the solid line represents the prediction calibration curve of the nomogram. The solid line has a closer fit to the ideal dotted line, which indicates better predictive accuracy of the nomogram $(p=0.22,0.74)$.

NPI has been reported as an important value for the prognostic evaluation and formulation of a treatment plan (26). A number of previous studies have shown that the ADC value of DWI, tumor volume doubling time of $3 \mathrm{D}$ ultrasound, and contralateral parenchymal enhancement of DCE-MRI could predict NPI (27-29). However, to the best of our knowledge, this study is the first attempt to combine DWI, IVIM, and DKI to predict the NPI of breast cancer. In our study, the IVIM parameter of $\mathrm{D}^{*}$ and the DKI parameter of MK are shown as independent predictors in the assessment of NPI. The prediction accuracy of combined $\mathrm{D}^{\star}$ and MK was higher than that of each single parameter. The pathological basis is that NPI can be used to reflect tumor proliferation and metastasis, which is associated with tumor cell heterogeneity, more microangiogenesis, higher blood volume, and vascularization. A higher grade of NPI manifests higher microperfusion and lower nonGaussian diffusivity.

Tumor cells with higher Ki-67 expression generally exhibit increasing heterogeneity and complexity of the microstructural level, thereby manifesting higher microperfusion and lower diffusivity (30). In this study, the $\mathrm{D}, \mathrm{D}^{*}, \mathrm{f}$, and $\mathrm{MK}$ values are independent predictors in discriminating the Ki-67 expression status. The AUC of the combined model demonstrated superior diagnostic performance compared with the single parameter, which is consistent with the study of Meng et al. (31, 32). However, in some other studies, it was observed that the expression of Ki-67 has no significant correlation with the D, MD, and MK values $(33,34)$. We speculated that this discrepancy might be related to the inclusion of lesions, the selection of b-values, and the ROI delineation.

Luminal A breast cancer is defined as a low-proliferation subtype and generally has a favorable prognosis compared with other subtypes. In this study, Luminal A showed the lowest $\mathrm{D}^{*}$ and $f$ values, indicating less intratumoral microperfusion. The reason may be that ER/PR expression is associated with the inhibition of angiogenesis, which would reduce perfusion (35). As ER-/PR-positive and higher Ki-67 indices tend to have lower diffusivity and vascularity, we found that Luminal B has the lowest D value. The HER-2-positive subtype demonstrated increased ADC values compared with the Luminal B subtype. The reason might be that HER-2 overexpression exhibits higher angiogenesis, which leads to an increased diffusion (36). The differentiation between the Luminal and non-Luminal tumors is of particular clinical importance since Luminal subtypes are treated with endocrine therapy and may benefit less from cytotoxic chemotherapy. In this study, non-Luminal breast cancer, including HER-2-positive and triple-negative tumors, had a higher $\mathrm{D}^{\star}$ value than Luminal A/B. It is likely that high vascularity in HER-2-positive and triple-negative tumors may overcome the restricted Gaussian diffusion related to high cellularity, which is in line with findings of Uslu et al. (37). On the other hand, TNBC has a poor response to endocrine or targeted therapy as well as chemotherapy, and the prognosis is worse than that of other subtypes (38). This study found that triple-negative tumors exhibited higher MK values than other subtypes, due to the most complex microstructure. Moreover, we demonstrated that $\mathrm{MK}$ could reflect the complexity of the microscopic structures in tissues more accurately, by using sufficiently high b-values of $2,000 \mathrm{~s} / \mathrm{mm}^{2}$ to eliminate the perfusion effect. In this study, the calibration curve for the combined genotype prediction models indicated that these models had good stability and that the corresponding nomograms could be helpful for visually and interpretatively predicting the genotypes of breast cancer patients.

The present research has some limitations. Firstly, there may be selection bias because of the relatively small sample size and the limited pathological types (most of which were invasive ductal carcinoma). Secondly, no unified standard exists for the option of number and value of $b$ used in IVIM and DKI scanning, and the repeatability of the b-value used in this 
study requires further verification. Thirdly, we simply calculated the ROI-based mean value of each parameter, which might not be enough to fully reflect the heterogeneity of tumors. An alternative processing method is to extract the whole volume of the lesions and analyze the histogram and texture features of each parameter map, which will be the focus of our future study.

In conclusion, the quantitative parameters of DWI, IVIM, and DKI are correlated with prognostic metrics. $\mathrm{D}^{\star}$ combined with $\mathrm{MK}$ is more valuable for assessing the Nottingham index. $\mathrm{ADC}, \mathrm{D}, \mathrm{D}^{*}, \mathrm{f}$, and $\mathrm{MK}$ are valuable for reflecting the KI-67 expression status. The AUC of combined $\mathrm{D}, \mathrm{D}^{\star}, \mathrm{MD}$, and $\mathrm{MK}$ could yield robust diagnostic performance for discriminating Luminal A/B from non-Luminal breast cancers. Combined MK and $\mathrm{f}$ can facilitate the diagnosis of triple-negative breast cancer. Therefore, this study suggests that the functional parameters of DWI, IVIM, and DKI may reveal clinical potential in the diagnosis of genotypes and prognostic factors and may contribute to opting for an appropriate therapeutic approach in the clinic.

\section{DATA AVAILABILITY STATEMENT}

The raw data supporting the conclusions of this article will be made available by the authors, without undue reservation.

\section{REFERENCES}

1. Torre LA, Siegel RL, Ward EM, Jemal A. Global Cancer Incidence and Mortality Rates and Trends-an Update. Cancer Epidemiol Biomarkers Prev (2016) 25:16-27. doi: 10.1158/1055-9965.EPI-15-0578

2. Amornsiripanitch N, Nguyen VT, Rahbar H, Hippe DS, Gadi VK, Rendi MH, et al. Diffusion-Weighted MRI Characteristics Associated With Prognostic Pathological Factors and Recurrence Risk in Invasive ER+/HER2- Breast Cancers. J Magn Reson Imaging (2018) 48:226-36. doi: 10.1002/jmri.25909

3. Roknsharifi S, Fishman MDC, Agarwal MD, Brook A, Kharbanda V, Dialani V. The Role of Diffusion Weighted Imaging as Supplement to Dynamic Contrast Enhanced Breast MRI: Can It Help Predict Malignancy, Histologic Grade and Recurrence? Acad Radiol (2019) 26:923-9. doi: 10.1016/j.acra

4. Rakha EA, El-Sayed ME, Menon S, Green AR, Lee AH, Ellis IO. Histologic Grading Is an Independent Prognostic Factor in Invasive Lobular Carcinoma of the Breast. Breast Cancer Res Treat (2008) 111:121-7. doi: 10.1007/s10549007-9768-4

5. Takahashi H, Oshi M, Asaoka M, Yan L, Endo I, Takabe K. Molecular Biological Features of Nottingham Histological Grade 3 Breast Cancers. Ann Surg Oncol (2020) 27:4475-85. doi: 10.1245/s10434-020-08608-1

6. Giuliano AE, Edge SB, Hortobagyi GN. Eighth Edition of the AJCC Cancer Staging Manual: Breast Cancer. Ann Surg Oncol (2018) 25:1783-5. doi: 10.1245/s10434-018-6486-6

7. Zhuang Z, Zhang Q, Zhang D, Cheng F, Suo S, Geng X, et al. Utility of Apparent Diffusion Coefficient as an Imaging Biomarker for Assessing the Proliferative Potential of Invasive Ductal Breast Cancer. Clin Radiol (2018) 73:473-8. doi: 10.1016/j.crad.2017.11.019

8. Zhang Y, Zhu Y, Zhang K, Liu Y, Cui J, Tao J, et al. Invasive Ductal Breast Cancer: Preoperative Predict Ki-67 Index Based on Radiomics of ADC Maps. Radiol Med (2020) 125:109-16. doi: 10.1007/s11547-019-01100-1

9. Chang RF, Chen HH, Chang YC, Huang CS, Chen JH, Lo CM. Quantification of Breast Tumor Heterogeneity for ER Status, HER2 Status, and TN Molecular Subtype Evaluation on DCE-MRI. Magn Reson Imaging (2016) 34:809-19. doi: 10.1016/j.mri.2016.03.001

\section{ETHICS STATEMENT}

The studies involving human participants were reviewed and approved by the institutional ethics committee of the affiliated hospital of Jining Medical University. The patients/participants provided their written informed consent to participate in this study.

\section{AUTHOR CONTRIBUTIONS}

WW, XZ, YC, and ZS contributed to the conception and design of the study. LZ and FZ organized the database. ZZ and WD performed the statistical analysis. WW wrote the first draft of the manuscript. WW and ZS contributed to manuscript revision. All authors read and approved the submitted version.

\section{FUNDING}

This work was supported by Medical and Health Science and Technology Development Project of Shandong Province (Grant number: 202009011151) and the Scientific Research Support Fund for Teachers of Jining Medical University (Grant number: JYFC2019FKJ088).

10. Shin JK, Kim JY. Dynamic Contrast-Enhanced and Diffusion-Weighted MR of Estrogen Receptor-Positive Invasive Breast Cancers: Associations Between Quantitative MR Parameters and Ki-67 Proliferation Status. J Magn Reson Imaging (2017) 45:94-102. doi: 10.1002/jmri.25348

11. Choi Y, Kim SH, Youn IK, Kang BJ, Park WC, Lee A. Rim Sign and Histogram Analysis of Apparent Diffusion Coefficient Values on Diffusion-Weighted MRI in Triple-Negative Breast Cancer: Comparison With ER-Positive Subtype. PloS One (2017) 12:e0177903. doi: 10.1371/journal.pone

12. He M, Ruan H, Ma M, Zhang Z. Application of Diffusion Weighted Imaging Techniques for Differentiating Benign and Malignant Breast Lesions. Front Oncol (2021) 11:694634. doi: 10.3389/fonc.2021.694634

13. Sumkin JH, Berg WA, Carter GJ, Bandos AI, Chough DM, Ganott MA, et al. Diagnostic Performance of MRI, Molecular Breast Imaging, and ContrastEnhanced Mammography in Women With Newly Diagnosed Breast Cancer. Radiology (2019) 293:531-40. doi: 10.1148/radiol.2019190887

14. Dorrius MD, Dijkstra H, Oudkerk M, Sijens PE. Effect of B Value and PreAdmission of Contrast on Diagnostic Accuracy of 1.5-T Breast DWI: A Systematic Review and Meta-Analysis. Eur Radiol (2014) 24:2835-47. doi: 10.1007/s00330-014-3338-z

15. Jensen JH, Helpern JA, Ramani A, Lu H, Kaczynski K. Diffusional Kurtosis Imaging: The Quantification of Non-Gaussian Water Diffusion by Means of Magnetic Resonance Imaging. Magn Reson Med (2005) 53:1432-40. doi: 10.1002/mrm.20508

16. Lecler A, Duron L, Zmuda M, Zuber K, Bergès O, Putterman M, et al. Intravoxel Incoherent Motion (IVIM) 3 T MRI for Orbital Lesion Characterization. Eur Radiol (2021) 31:14-23. doi: 10.1007/s00330-020-07103-1

17. Horvat JV, Bernard-Davila B, Helbich TH, Zhang M, Morris EA, Thakur SB, et al. Diffusion-Weighted Imaging (DWI) With Apparent Diffusion Coefficient (ADC) Mapping as a Quantitative Imaging Biomarker for Prediction of Immunohistochemical Receptor Status, Proliferation Rate, and Molecular Subtypes of Breast Cancer. J Magn Reson Imaging (2019) 50:83646. doi: 10.1002/jmri.26697

18. Kim Y, Ko K, Kim D, Min C, Kim SG, Joo J, et al. Intravoxel Incoherent Motion Diffusion-Weighted MR Imaging of Breast Cancer: Association With 
Histopathological Features and Subtypes. Br J Radiol (2016) 89:20160140. doi: 10.1259/bjr.20160140

19. Huang Y, Lin Y, Hu W, Ma C, Lin W, Wang Z, et al. Diffusion Kurtosis at 3.0T as an In Vivo Imaging Marker for Breast Cancer Characterization: Correlation With Prognostic Factors. J Magn Reson Imaging (2019) 49:845-56. doi: 10.1002/jmri.26249

20. Zhang Q, Ouyang H, Ye F, Song Y, Xie L, Zhao X, et al. Feasibility of Intravoxel Incoherent Motion Diffusion-Weighted Imaging in Distinguishing Adenocarcinoma Originated From Uterine Corpus or Cervix. Abdom Radiol (NY) (2021) 46:732-44. doi: 10.1007/s00261-020-02586-4

21. Kawashima H, Miyati T, Ohno N, Ohno M, Inokuchi M, Ikeda H, et al. Differentiation Between Luminal-A and Luminal-B Breast Cancer Using Intravoxel Incoherent Motion and Dynamic Contrast-Enhanced Magnetic Resonance Imaging. Acad Radiol (2017) 24:1575-81. doi: 10.1016/ j.acra.2017.06.016

22. Mao W, Ding Y, Ding X, Wang Y, Fu C, Zeng M, et al. Pathological Assessment of Chronic Kidney Disease With DWI: Is There an Added Value for Diffusion Kurtosis Imaging? J Magn Reson Imaging (2021) 54:508-17. doi: 10.1002/jmri.27569

23. Galea MH, Blamey RW, Elston CE, Ellis IO. The Nottingham Prognostic Index in Primary Breast Cancer. Breast Cancer Res Treat (1992) 22:207-19. doi: $10.1007 / \mathrm{BF} 01840834$

24. Zhao M, Fu K, Zhang L, Guo W, Wu Q, Bai X, et al. Intravoxel Incoherent Motion Magnetic Resonance Imaging for Breast Cancer: A Comparison With Benign Lesions and Evaluation of Heterogeneity in Different Tumor Regions With Prognostic Factors and Molecular Classification. Oncol Lett (2018) 16:5100-12. doi: 10.3892/ol.2018.9312

25. Yang ZL, Li Y, Zhan CA, Hu YQ, Guo YH, Xia LM, et al. Evaluation of Suspicious Breast Lesions With Diffusion Kurtosis MR Imaging and Connection With Prognostic Factors. Eur J Radiol (2021) 145:110014. doi: 10.1016/j.ejrad.2021.110014

26. Tan PH, Ellis I, Allison K, Brogi E, Fox SB, Lakhani S, et al. The 2019 World Health Organization Classification of Tumours of the Breast. Histopathology (2020) 77:181-5. doi: 10.1111/his.14091

27. Cipolla V, Santucci D, Guerrieri D, Drudi FM, Meggiorini ML, de Felice C. Correlation Between 3T Apparent Diffusion Coefficient Values and Grading of Invasive Breast Carcinoma. Eur J Radiol (2014) 83:2144-50. doi: 10.1016/ j.ejrad.2014.09.015

28. Zhang S, Ding Y, Zhou Q, Wang C, Wu P, Dong J. Correlation Factors Analysis of Breast Cancer Tumor Volume Doubling Time Measured by 3D-Ultrasound. Med Sci Moni (2017) 23:3147-53. doi: 10.12659/ msm.901566

29. van der Velden BHM, Elias SG, Bismeijer T, Loo CE, Viergever MA, Wessels LFA, et al. Complementary Value of Contralateral Parenchymal Enhancement on DCE-MRI to Prognostic Models and Molecular Assays in High-Risk ERp/ HER2 Breast Cancer. Clin Cancer Res (2017) 23:6505-15. doi: 10.1158/10780432.CCR-17-0176

30. Shen L, Zhou G, Tong T, Tang F, Lin Y, Zhou J, et al. ADC at 3.0T as a Noninvasive Biomarker for Preoperative Prediction of Ki67 Expression in Invasive Ductal Carcinoma of Breast. Clin Imaging (2018) 52:16-22. doi: 10.1016/j.clinimag.2018.02.010

31. Meng N, Wang X, Sun J, Han D, Bai Y, Wei W, et al. A Comparative Study of the Value of Amide Proton Transfer-Weighted Imaging and Diffusion
Kurtosis Imaging in the Diagnosis and Evaluation of Breast Cancer. Eur Radiol (2021) 31:1707-17. doi: 10.1007/s00330-020-07169-x

32. Meng N, Wang XJ, Sun J, Huang L, Wang Z, Wang KY, et al. Comparative Study of Amide Proton Transfer-Weighted Imaging and Intravoxel Incoherent Motion Imaging in Breast Cancer Diagnosis and Evaluation. J Magn Reson Imaging (2020) 52:1175-86. doi: 10.1002/ jmri.27190

33. Suo S, Cheng F, Cao M, Kang J, Wang M, Hua J, et al. Multiparametric Diffusion-Weighted Imaging in Breast Lesions: Association With Pathologic Diagnosis and Prognostic Factors. J Magn Reson Imaging (2017) 46:740-50. doi: $10.1002 /$ jmri.25612

34. Song SE, Cho KR, Seo BK, Woo OH, Park KH, Son YH, et al. Intravoxel Incoherent Motion Diffusion Weighted MRI of Invasive Breast Cancer: Correlation With Prognostic Factors and Kinetic Features Acquired With Computer-Aided Diagnosis. J Magn Reson Imaging (2019) 49:118-30. doi: 10.1002/jmri.26221

35. Iima M, Kataoka M, Kanao S, Onishi N, Kawai M, Ohashi A, et al. Intravoxel Incoherent Motion and Quantitative Non-Gaussian Diffusion MR Imaging: Evaluation of the Diagnostic and Prognostic Value of Several Markers of Malignant and Benign Breast Lesions. Radiology (2018) 287:432-41. doi: 10.1148/radiol.2017162853

36. Suo S, Zhang D, Cheng F, Cao M, Hua J, Lu J, et al. Added Value of Mean and Entropy of Apparent Diffusion Coefficient Values for Evaluating Histologic Phenotypes of Invasive Ductal Breast Cancer With MR Imaging. Eur Radiol (2019) 29:1425-34. doi: 10.1007/s00330-018-5667-9

37. Uslu H, Önal T, Tosun M, Arslan AS, Ciftci E, Utkan NZ. Intravoxel Incoherent Motion Magnetic Resonance Imaging for Breast Cancer: A Comparison With Molecular Subtypes and Histological Grades. Magn Reson Imaging (2021) 78:35-41. doi: 10.1016/j.mri.2021.02.005

38. Ma Y, Shan D, Wei J, Chen A. Application of Intravoxel Incoherent Motion Diffusion-Weighted Imaging in Differential Diagnosis and Molecular Subtype Analysis of Breast Cancer. Am J Transl Res (2021) 13:3034-43. https://www. ncbi.nlm.nih.gov/pmc/articles/PMC8129298/.

\section{Conflict of Interest: Author WD is employed by GE Healthcare.}

The remaining authors declare that the research was conducted in the absence of any commercial or financial relationships that could be construed as a potential conflict of interest.

Publisher's Note: All claims expressed in this article are solely those of the authors and do not necessarily represent those of their affiliated organizations, or those of the publisher, the editors and the reviewers. Any product that may be evaluated in this article, or claim that may be made by its manufacturer, is not guaranteed or endorsed by the publisher.

Copyright (c) 2022 Wang, Zhang, Zhu, Chen, Dou, Zhao, Zhou and Sun. This is an open-access article distributed under the terms of the Creative Commons Attribution License (CC BY). The use, distribution or reproduction in other forums is permitted, provided the original author(s) and the copyright owner(s) are credited and that the original publication in this journal is cited, in accordance with accepted academic practice. No use, distribution or reproduction is permitted which does not comply with these terms. 